\title{
A Finite Element Study of Crack Behavior for Carbon Nanotube Reinforced Bone Cement
}

\author{
Kaveh PourAkbar Saffar ${ }^{1 *}$, Ahmad Raeisi Najafi ${ }^{2 *}$, Manssour H. Moeinzadeh ${ }^{3}$, Leszek J. Sudak ${ }^{1}$ \\ ${ }^{1}$ Department of Mechanical and Manufacturing Engineering, University of Calgary, Calgary, Canada \\ ${ }^{2}$ Department of Mechanical Science and Engineering, University of Illinois at Urbana-Champaign, Urbana, USA \\ ${ }^{3}$ Department of Industrial and Enterprise Systems Engineering, University of Illinois at Urbana-Champaign, Urbana, USA \\ Email: kpourakb@ucalgary.ca, arnajafi@illinois.edu, manssour@illinois.edu, lsudak@ucalgary.ca
}

Received May 15, 2013; revised June 15, 2013; accepted June 23, 2013

Copyright (c) 2013 Kaveh PourAkbar Saffar et al. This is an open access article distributed under the Creative Commons Attribution License, which permits unrestricted use, distribution, and reproduction in any medium, provided the original work is properly cited.

\begin{abstract}
Polymethylmethacrylate (PMMA) bone cement is a polymeric material that is widely used as a structural orthopedic material. However, it is not an ideal material for bone grafting due to its fragility. Carbon nanotubes (CNTs) have been introduced in order to reinforce PMMA resulting in a composite material which exhibits improved tensile properties, increased fatigue resistance and fracture toughness. This improvement is potentially due to bridging and arresting cracks as well as absorption of energy. In this study, a two-dimensional finite element model is presented for the fracture analysis of PMMA-CNT composite material. Instead of the classical single fiber model, the present work considers an ensemble of CNTs interacting with a pre-existing crack. Casca is used to produce a two dimensional mesh and the fracture analysis is performed using Franc 2D. The model is subjected to uni-axial loading in the transverse plane and the interaction between the crack and CNTs is evaluated by determining the stress intensity factor in the vicinity of the crack tips. The effects of geometric parameters of the CNTs and the material structural heterogeneity on crack propagation trajectory are investigated. Furthermore, the effects of CNT diameter, wall thickness and elastic mismatch between the matrix and the nanotubes on crack growth are studied. The results illustrate that the CNTs repel cracks during loading as they act as barriers to crack growth. As a result, the incorporation of CNTs into PMMA reduces crack growth but more importantly increases the fracture resistance of bone cement.
\end{abstract}

Keywords: Bone Cement; Carbon Nanotube; Finite Element; Crack Propagation; Stress Intensity Factor

\section{Introduction}

Polymethylmethacrylate (PMMA) bone cement is a polymeric material that shows good compatibility with living tissues. It is commonly used as bone filling cement during orthopedic surgeries, such as total joint anthroplasty $[1,2]$. Despite its excellent performance, several failure mechanisms of cemented prosthetics have been well documented [3-5].

It is well-known that both biological and mechanical factors (such as mechanical loading, debonding at the cement-prosthesis interface, fatigue failure and cracks in the cement mantle) contribute to the failure of total hip arthroplasty; however, it is the mechanical processes that are considered to be the dominant feature leading to aseptic loosening [6,7]. In particular, it is well accepted that cracks exist in the cement mantle. These cracks

${ }^{*}$ Authors with equal contribution. typically are generated from polymerization of the PMMA from natural pores formed in the mantle during the curing process or they propagate into the cement mantle from the failure of the cement/implant interface [8]. For example, McCormack \& Prendergast [9] experimentally investigated the amount of damage accumulation that occurs in the cement mantle under flexural loading. Other studies $[6,10]$ have demonstrated that failure of the cement mantle, due to the presence of radial cracks, initiated loosening of the prosthetic. Thus, the fracture of PMMA bone cement is a critical factor affecting implant failure.

There have been numerous studies carried out to improve the mechanical and physical behavior of bone cement by incorporating additive materials as reinforcing phases to the bone cement $[5,11]$. However, limited success has been achieved mainly due to filler-damage scale mismatch, as the size of the reinforcing agents is larger than the scale of fatigue damage. This makes the rein- 
forcement ineffective against crack propagation and damage accumulation [5].

Nano-structured materials have generated considerable interest in the literature mainly because of their potential for large improvements in the mechanical behavior as compared to traditional structural materials. In particular, extensive research has shown that carbon nanotubes (CNTs) exhibit exceptional mechanical and chemical properties as well as thermal stability. For example, in composite science research, the use of CNTs as a reinforcing agent makes them ideal candidates for producing a new generation of nano-composites [12-14]. More recently, in the area of bioengineering, CNTs have been used as biocompatible and supportive substrates $[15,16]$. In particular, CNTs have been considered for effective employment in bone tissue engineering either as a scaffold for bone material regeneration and growth $[17,18]$, as reinforcement phase for ceramic artificial bone such as hydroxyapatite [19-21] and polymeric bone fillers, such as PMMA [3-5,22]. More importantly, the dimensional incompatibility between the reinforcing fibers and the size of fatigue damage is no longer an issue. In other words, unlike the large size of conventional fibers (on the order of microns), the small size of CNTs (order of nanometers) is comparable to the scale of fatigue damage found in the bone cement. Consequently, the use of CNTs as a reinforcement phase in PMMA can have a significant benefit in preventing crack growth with the overall goal of reducing implant motion and revision surgery. The enhancement of the fatigue performance of bone cement is a result of the CNT's high surface area to volume ratio which increases the physical interface and improves the quality of stress transfer between the constituent phases.

The use of experimentation, simulation and modeling in the study of nano-scale materials such as CNTs on the structural response (such as fracture characterization) is still in its infancy. The small size of the components of nano-composites makes it difficult to clearly observe every factor incorporated with the real failure mechanisms in experiments. So, controlled experiments at the nano-scale are difficult. The use of molecular dynamics simulations remains expensive and formidable especially in the case of large scale systems. For example, the size of atomistic systems cannot exceed billions of particles; however, realistic systems contain $10^{23}$ atoms and more. Continuum mechanics approaches based on the finite element method have been used extensively to evaluate the elastic properties of CNT-based composites. For example, Gawandi and colleagues addressed the problem of a single nano-fiber interacting with a crack $[23,24]$. However, these works are limited because the atomistic structure of the nanotube has been neglected in the formulation of the finite element model. It should be noted that additional, supplementary works on crack-fiber interaction can be found in the following works: [25-35]. However, the study of the interaction problem between a crack and an ensemble of multiple CNTs remains absent from the literature. Thus, in the present work we develop a two dimensional finite element model incorporating an array of CNTs as the reinforcing phase in PMMA bone cement where the atomistic structure of CNTs are taken into account. The results clearly illustrate that CNTs repel cracks during loading as they act as barriers to crack growth. Moreover, it is observed that the incorporation of CNTs into PMMA reduces crack growth but more importantly increases the fracture resistance of bone cement.

\section{Problem Statement}

We consider a two dimensional setting in which single walled carbon nanotubes (SWCNTs) are assumed to be well dispersed and aligned parallel to one another and embedded within the PMMA bone cement, (referred to as the matrix). A crack is situated in the matrix and the loading on the configuration is uni-axial tension applied perpendicular to the $X$ axis, as shown (see Figure 1). Crack extension takes place in the plane of the nanotube cross section and plane strain conditions are assumed. It is reasonable to assume that the matrix and CNT is isotropic and linear elastic subject to small deformations [36]. In addition, all boundaries between materials are assumed to have continuous displacements and tractions.

The SWCNT, from the structural point of view, can be thought of as a graphene sheet rolled up into a cylindrical

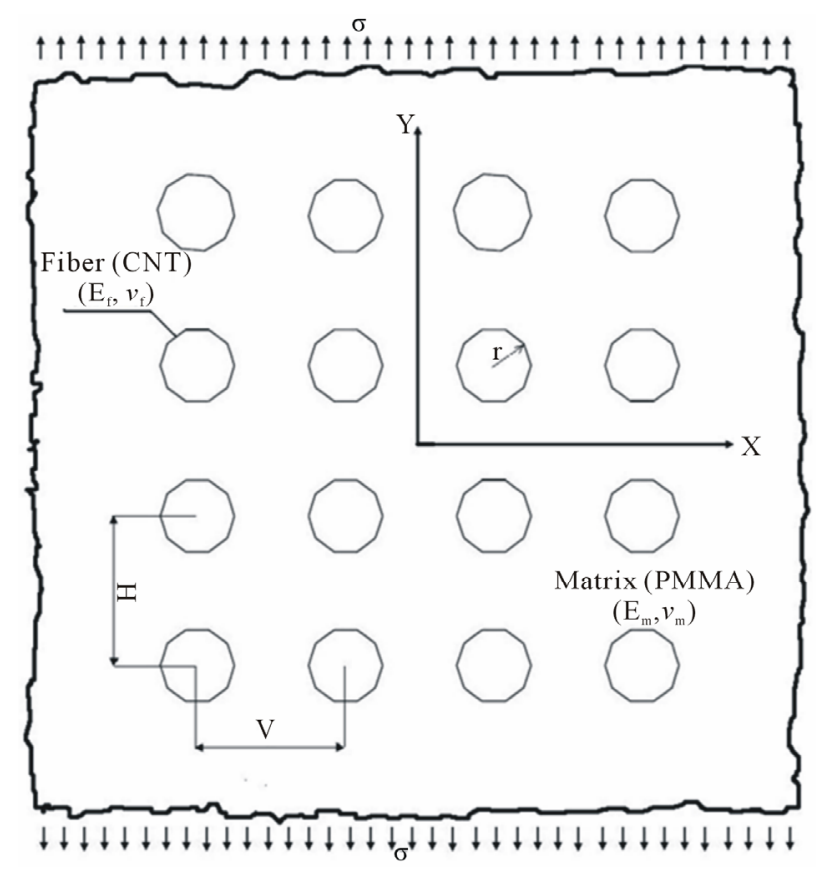

Figure 1. 2D model of CNT reinforced bone cement. 
shape with a diameter on the order of nanometers. In addition, the efficiency of nanotube reinforcement depends on the morphology and distribution of CNTs. This requires that the atomistic structure of CNTs be taken into account when formulating the finite element model. In view of this, the development of our model requires that the structurally discrete CNTs be replaced by an equivalent effective solid fiber continuum phase whose length and diameter are kept the same as those of the CNT so as to preserve the small-scale features of the nanotube (see Figure 2). Assuming isostrain conditions, the modulus of elasticity and Poisson's ratio for the effective solid fiber are readily determined from the CNT dimensions and given by Equation (1),

$$
E_{f}=\frac{r^{2}-(r-t)^{2}}{r^{2}} E_{C N T}
$$

where $r, t$, and $E_{C N T}$ are the radius, wall thickness and elastic stiffness of the CNT, respectively.

The model considers that the CNTs have a diameter of $1.5 \mathrm{~nm}$, a wall thickness of $0.34 \mathrm{~nm}$ and elastic modulus of $0.97 \mathrm{TPa}$ [37]. The PMMA is defined by two elastic constants: elastic stiffness $E_{m}=2.5 \mathrm{GPa}$ and Poisson's ratio $v_{m}=0.35$. Using (1) the properties for the effective solid fiber continuum phase are found to be $E_{f}=680 \mathrm{GPa}$ and $v_{f}=0.28$.

\section{Finite Element Model}

The 2D finite element mesh is built using Casca [38] and Franc 2D [39] is used for the fracture analysis of the model. Triangular elements with six nodes comprising 12 degrees of freedom are used to produce the finite element mesh structure. In order to avoid boundary effects in the results, the model dimensions are taken sufficiently large compared to the CNT diameter and crack length (see Figure 3). It should be noted that our simulations have been tested and verified against the analytical solution obtained from the model illustrated in Figure 4(a).

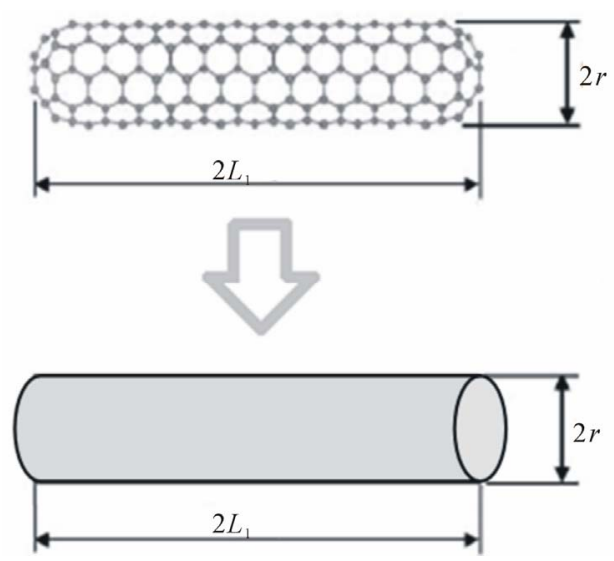

Figure 2. Nanotube as an effective fiber.
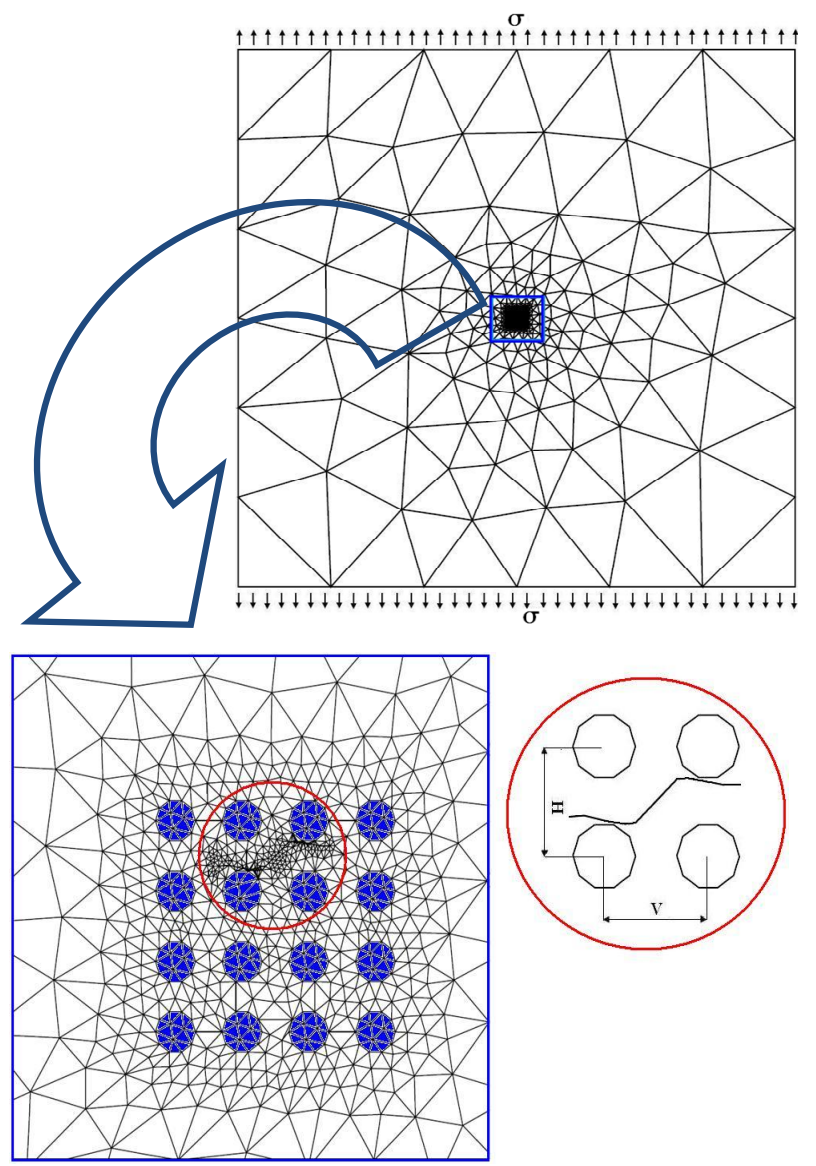

Figure 3. Finite element model of crack-CNTs interaction.

In an effort to understand how the number, location, configuration, and material characteristics of CNTs influences' the variation of the stress intensity factor (SIF) at the crack tips as well as crack trajectory, finite element simulations for two problems is investigated. In the first problem we will consider three different configurations of crack-CNT interaction. The first configuration consists of a single CNT with $1.5 \mathrm{~nm}$ diameter placed in a sufficiently large matrix subjected to a uni-axial stress, $\sigma$, while having an internal crack of length $2 L=1.5 \mathrm{~nm}$ placed in a horizontal orientation to the external load as shown in Figure 4(a). A second configuration consisting of three CNTs placed on a cross arrangement with an internal crack placed in-between the CNTs in a horizontal orientation to the externally applied load (see Figure 4(b)).

The third configuration considers the mixed mode (modes I and II) problem for a variable inclined crack in the PMMA matrix located in the middle of a square arrangement of CNT as shown in Figure $5(H=V=3 \mathrm{~nm}$, $2 L=1.84 \mathrm{~nm}$ ). This illustrates the effect of changing the crack orientation on the variation of the SIF at crack tips. Now, with respect to the second problem, let us consider the effects of crack propagation. The model includes dif- 


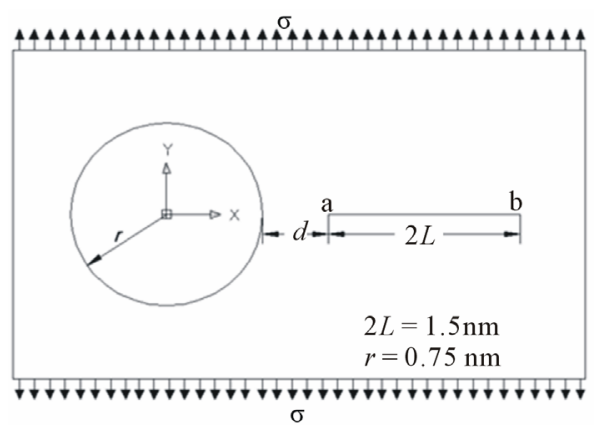

(a)

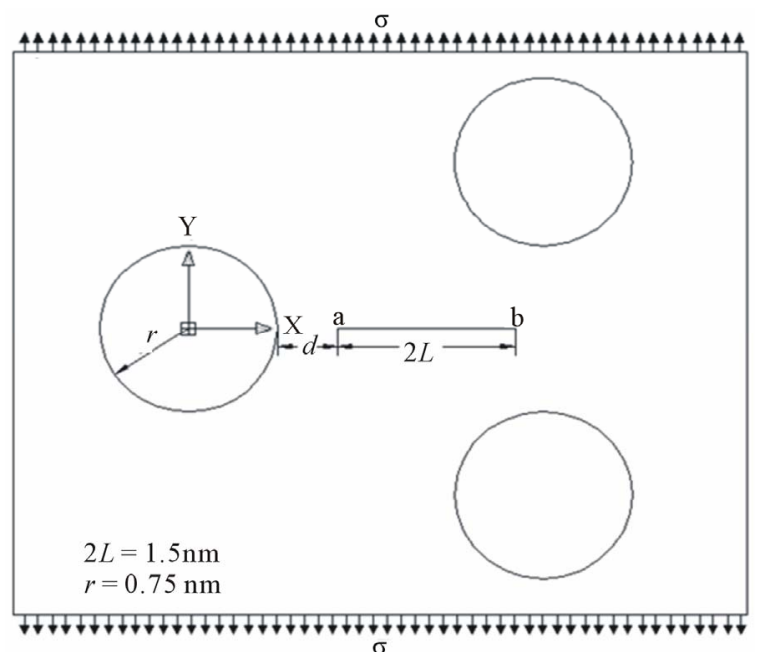

(b)

Figure 4. Two configurations for mode-I SIF analysis.

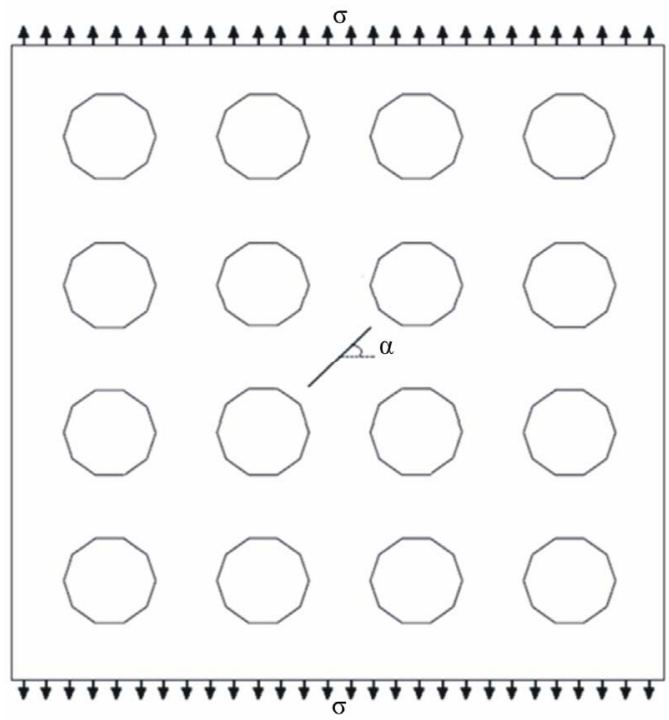

Figure 5. A crack with angle $\alpha$ oriented in the middle of a square arrangement of CNTs in the matrix.

ferent arrangement of CNTs embedded into the PMMA matrix (see Figures 6-8, respectively). Here, the matrix dimensions are much larger than the interested area. So

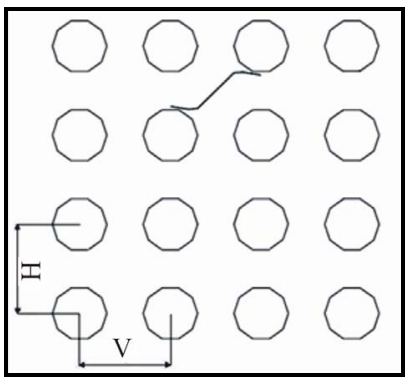

(a)

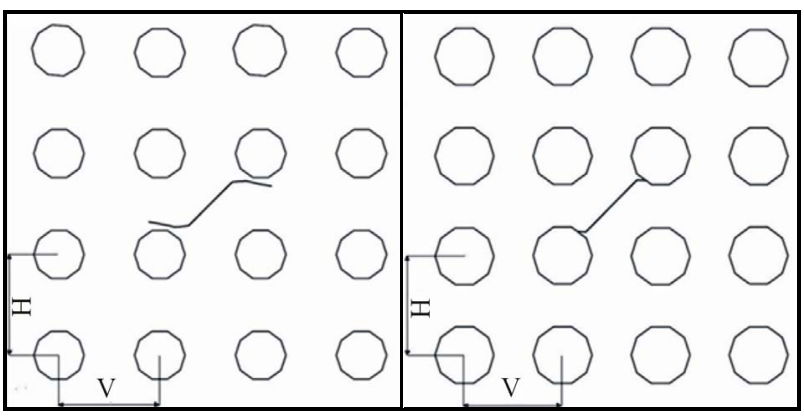

(b)

(c)

Figure 6. Finite Element Model of Crack-CNTs interaction. The model includes fibers with $2 \mathrm{r}=1.5 \mathrm{~nm}, \mathrm{Ef}=0.68 \mathrm{TPa}$, $\mathrm{Em}=2.5 \mathrm{GPa}$. (a) $\mathrm{H}=\mathrm{V}=\mathbf{2 . 5} \mathrm{nm}$ and primary crack length $1.42 \mathrm{~nm}$; (b) $\mathrm{H}=\mathrm{V}=3 \mathrm{~nm}$ and primary crack length 1.84 $\mathrm{nm}$; (c) $\mathrm{H}=\mathrm{V}=2.5 \mathrm{~nm}$ and primary crack length $1.84 \mathrm{~nm}$.

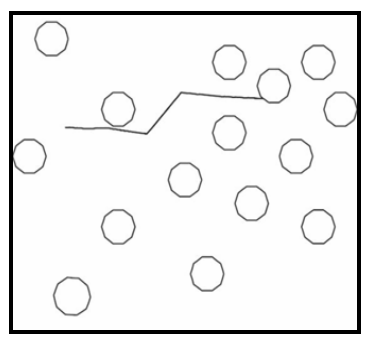

(a)

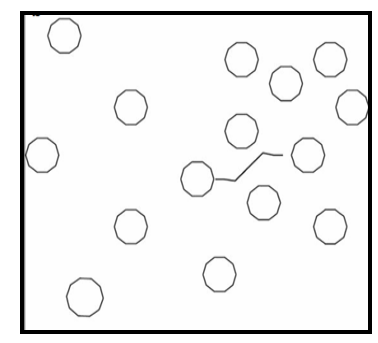

(b)
Figure 7. Crack propagation is severely affected by the separation of the fibers.

there are no boundary effects in the results. Uniaxial loading normal to the boundary is taken and the crack propagation trajectory is obtained. Using Franc 2D, the direction of crack propagation is determined by the application of the maximum hoop stress. The hoop stress $\left(\sigma_{\theta}\right)$ is determined around the crack tip on the circumference of a constant radius circle. Thus, crack propagation is in the direction of the maximum hoop stress. The corresponding mathematical description is provided by expressions (2) and (3).

$$
\begin{aligned}
& \frac{\partial \sigma_{\theta}}{\partial \theta}=0 \\
& \frac{\partial^{2} \sigma_{\theta}}{\partial \theta^{2}}<0
\end{aligned}
$$




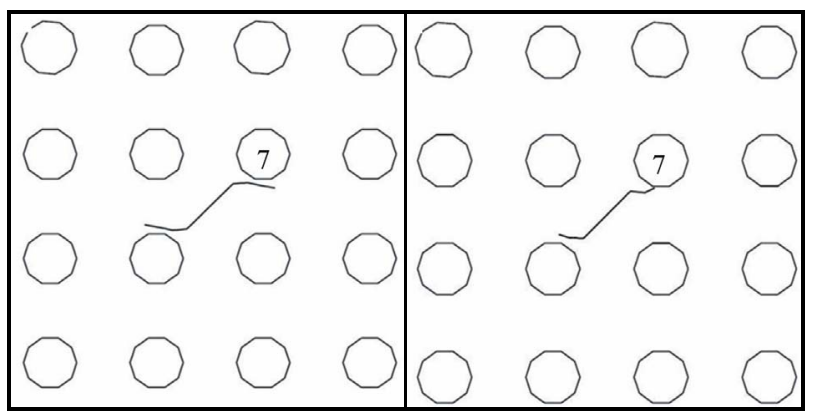

(a)

(b)

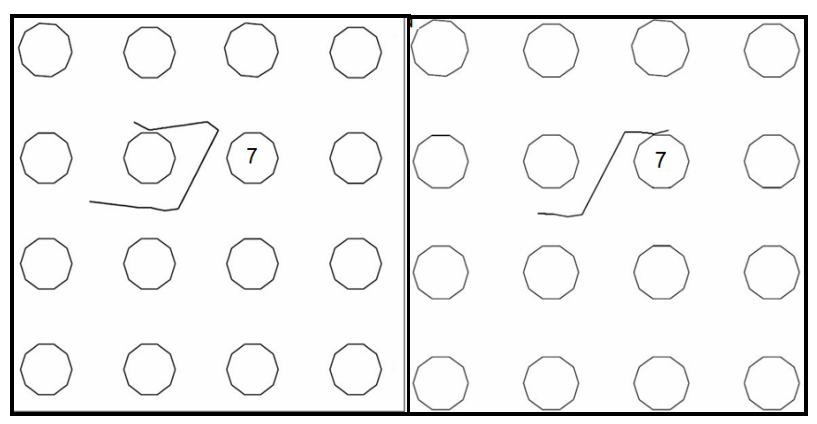

(c)

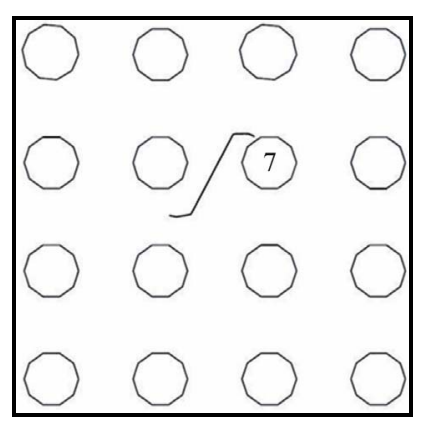

(e)

Figure 8. Crack propagation trajectory is affected by fiber material properties. Stiff fiber repels the crack where soft fiber or hole leads fiber attracting the crack. (a) $\mathbf{E f}=\mathbf{E f 7}=$ $0.68 \mathrm{TPa}$; (b) $\mathrm{Ef}=0.68 \mathrm{TPa}$, and a hole instead of fiber number 7; (c) Ef $=$ Ef7 $=0.68 \mathrm{TPa}$; (d) Ef $=0.68 \mathrm{TPa}, \mathrm{Ef} 7=$ $0.2 \mathrm{TPa}$; (e) Ef =0.68 TPa, and a hole instead of fiber 7 .

\section{Results and Discussion}

Figures 9(a) and (b) show the normalized SIF versus crack location with respect to the arrangement of CNTs. The crack location is considered as the normalized distance of the crack from the single CNT shown in Figure 4(a) (corresponding to Figure 9(a)), and the normalized distance of the crack from the CNT at left of the cross arrangement as shown in Figure 4(b) (corresponding to Figure 9(b)). The simulation results indicate that as the crack distance from the CNT increases the SIF also increases for both arrangements. In fact, at close distances, the CNT (on the left) has a shielding effect. Figure 9(c) shows the SIF values for both arrangements which give an idea on the effect of the presence of two extra CNTs

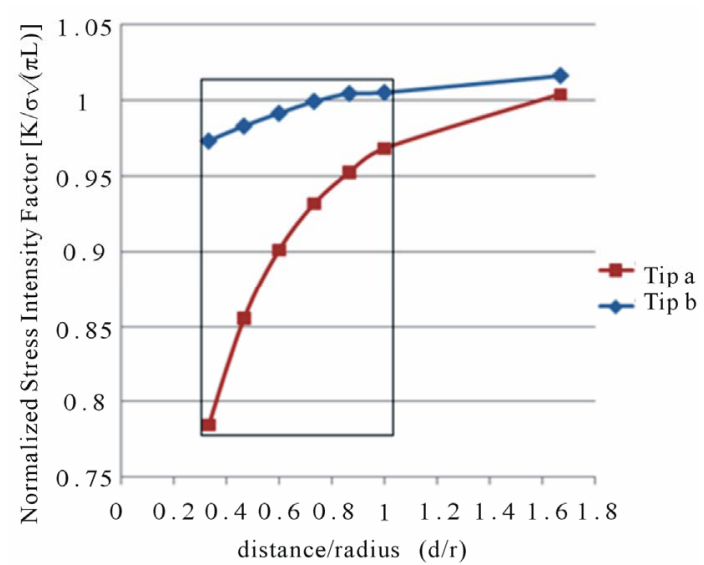

(a)

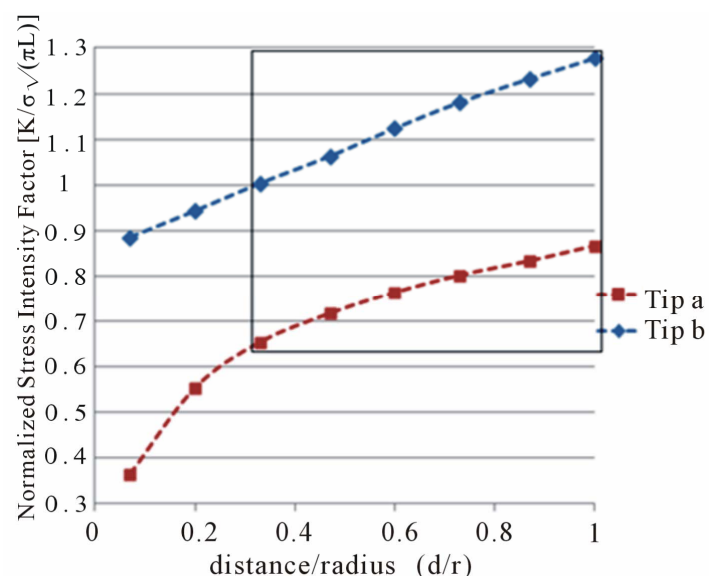

(b)

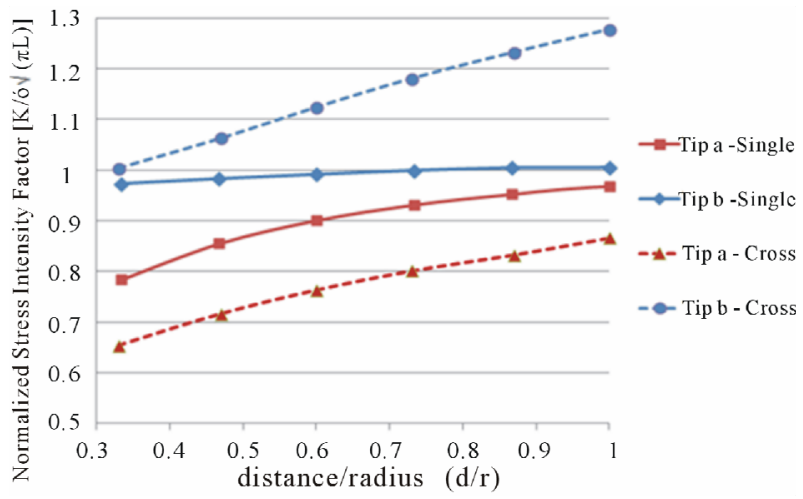

(c)

Figure 9. Normalized SIF of crack tips versus the normalized distance from the fiber at left $(d / r)$ for two configurations: (a) Single CNT; (b) Cross arrangement of three CNTs; and (c) Both arrangements altogether.

in the cross arrangement in comparison with the single CNT arrangement. According to Figure 9(c), the cross arrangement leads to significant stress shielding at crack tip $a$, while, at crack tip $b$ we observe a dramatic stress amplification. This implies that the presence of two extra CNTs in a cross arrangement pattern (at right) is effect- 
tive as a reinforcement in regions close enough to the other CNT which faces crack tip $a$ while the crack is actually encouraged to grow from crack tip $b$ due to the presence of CNTs at right. Obviously, this effect is dependent on the separation of the two CNTs at right (i.e. the crack is encouraged to grow from crack tip $b$ and pass through the space between them if this separation is more than a certain amount). Close arrangements of CNTs would definitely have a barrier effect against crack growth. This will be discussed later.

Figure 10 shows the values of modes I and II normalized SIF for the lower-left tip of the crack in the model shown in Figure 5, with respect to varying $\alpha$. Due to the symmetry of the problem close values with the same trend are expected for the other crack tip.

The model which is developed for crack propagation analysis is shown in Figure 6. The model includes an array of CNTs and an internal crack (at an angle of 45 degrees). The crack propagation simulation shows that crack propagation trajectory is severely affected by the arrangement and material properties of the CNTs. The effect of the CNT arrangement is presented in Figures 6(a)-(c), respectively. The results show that the crack is repelled by the CNTs and the crack follows a trajectory between them when the distance between the CNTs is more than a certain amount. In fact, the crack deviates and does not enter the CNTs. On the other hand, if CNTs are situated closer together, the crack is not able to propagate through the space between the CNTs. In this situation, the CNTs act as a barrier to crack growth.

Normalized SIF values for modes I and II ( $K_{I}$ and $\left.K_{I I}\right)$ at the crack tips for the arrangements shown in Figure 6 are also presented in Table 1. At first, proximity of the values for crack tips $a$ and $b$ is observed which is due to the problem symmetry. A comparison between the second and third columns of this table provides sufficient

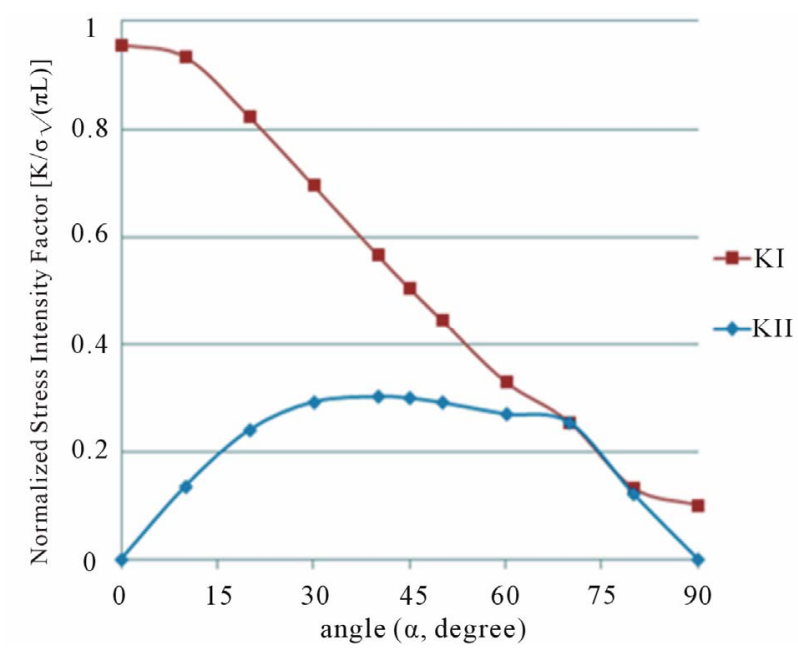

Figure 10. Normalized SIF of a crack with changing angle in the middle of square arrangement of CNTs.
Table 1. Normalized SIF values of crack tips for models shown in Figure 8.

\begin{tabular}{cccc}
\hline & $\begin{array}{c}H=V=\mathbf{2 . 5} \mathbf{~ n m} \\
\mathbf{2 L}=\mathbf{1 . 4 2} \mathbf{~ n m}\end{array}$ & $\begin{array}{c}H=V=\mathbf{3 m} \mathbf{~ n m} \\
\mathbf{2 L}=\mathbf{1 . 8 4} \mathbf{~ n m}\end{array}$ & $\begin{array}{c}H=V=\mathbf{2 . 5} \mathbf{~ n m} \\
\mathbf{2 L}=\mathbf{1 . 8 4} \mathbf{~ n m}\end{array}$ \\
\hline KI-tip $\boldsymbol{a}$ & 0.402 & 0.454 & 0.353 \\
KII-tip $\boldsymbol{a}$ & 0.223 & 0.272 & 0.253 \\
KI-tip $b$ & 0.411 & 0.457 & 0.355 \\
KII-tip $b$ & 0.220 & 0.272 & 0.254 \\
\hline
\end{tabular}

evidence as to why the crack grows in the space between the distanced CNTs (e.g. Figure 6(b)), while it stops between close CNTs (e.g. Figure 6(c)).

In the case of randomly dispersed CNTs, dispersion of the CNTs has a significant influence on the material properties of CNT reinforced polymer matrix nano-composites [40]. In view of this, crack propagation in a randomly dispersed matrix of CNTs is illustrated in Figures 7(a) and (b). These figures clearly show that crack propagation trajectory is severely affected by the separation of the CNTs. The crack appears to select the path of least resistance between the CNTs for propagation where the local CNT density is low, whereas crack growth slows or even comes to a complete stop when the local CNT density is high (i.e., the distance between the CNTs are small).

Figure 8 shows the effect of material properties on crack propagation trajectory. In this model, the elastic modulus of one of the CNTs (i.e., $\mathrm{CNT}_{7}$ ) is different from the others. Different values are assumed for $\mathrm{CNT}_{7}$ and the effect of this variation upon crack propagation is shown. Here, the elastic modulus of the CNT has a value of $E_{f}=E_{f 7}=0.68 \mathrm{TPa}$ (Figures 8(a) and (c)), $E_{f}=0.68$ $\mathrm{TPa}$ and $E_{f 7}=0.2 \mathrm{TPa}$ (Figure 8(d)). In Figures 8(b) and (e) we assume a hole instead of a $\mathrm{CNT}_{7}\left(E_{f 7}=0 \mathrm{TPa}\right)$ and the elastic modulus of the rest of CNTs is $E_{f}=0.68 \mathrm{TPa}$. It appears that the stiff CNT repels the crack whereas the soft CNT or hole leads to attracting the crack.

Table 2 presents the normalized SIF values for the crack tips corresponding to the different cases as illustrated in Figure 8. These values justify the tendency of the crack to grow along crack tips $a$ and $b$, when the location of the crack and the stiffness of $\mathrm{CNT}_{7}$ is changed. It is interesting to note that the SIF values and crack propagation trajectories were also evaluated for the case when the CNT stiffness was assumed to be $E_{f}=0.8 \mathrm{TPA}$ instead of 0.68 TPA. No significant changes were observed which might suggest that the behavior of the crack, instead of the separation and arrangement of the CNTs, is affected by the stiffness mismatch between the CNT and the matrix rather than the slight deviation of the material property of the CNT inclusions itself.

In general, the results of this study describe the overall improvement of the resistance of the PMMA bone ce ment to fracture that has been observed experimentally 
Table 2. Normalized SIF values of crack tips for models shown in Figure 10.

\begin{tabular}{cccccc}
\hline Figure \# & $10 \mathrm{a}$ & $10 \mathrm{~b}$ & $10 \mathrm{c}$ & $10 \mathrm{~d}$ & $10 \mathrm{e}$ \\
\hline KI—tip $a$ & 0.454 & 0.626 & 0.110 & 0.111 & 0.304 \\
KII-tip $a$ & 0.272 & 0.549 & 0.340 & 0.340 & 0.467 \\
KI-tip $b$ & 0.457 & 0.529 & 0.252 & 0.252 & 0.332 \\
KII-tip $b$ & 0.272 & 0.401 & 0.299 & 0.299 & 0.323 \\
\hline
\end{tabular}

$[3-5,22]$ and the significant clinical applications that can be achieved. However, it must be noted that a number of simplifying assumptions have been made. For example, the aligned configuration of the CNTs in the PMMA matrix, as assumed in this study, cannot be easily achieved. This would require the application of various special methods and processes which would undoubtedly make a CNT-PMMA bone cement practically unattractive. Consequently, even though randomly oriented configuration of CNTs leads to less effective improvement of the mechanical properties of the resulting nano-composite [40], the need to create more efficient cements for joint arthroplasty is clinically necessary. Also we have neglected the relatively weak non-bonding interactions and the van der Waals forces between the CNTs. However, by choosing a continuum medium for the CNT and the PMMA matrix, it is assumed that such interactions do not affect the crack propagation process significantly. Finally, although the imperfect bonding model between matrix and inclusion may lead to an increase in the SIF [41], the classical assumption of perfect bonding can still be considered valid as long as the CNT aspect ratio is taken to be large enough [19].

\section{Conclusion}

This work presents a finite element study of the fracture behavior of CNT reinforced bone cement through the adoption of a simplified model. It is found that the material properties and the morphological parameters of CNTs greatly influence the fracture behavior. The results have shown that the effect of CNTs on the crack could lead to a decrease in the SIF at the crack tips. However, this effect is limited to the vicinity of the CNTs. The crack growth trajectory is also influenced by the nanostructural arrangement of the components of the composite. More importantly, the results clearly illustrate that CNTs could either repel or attract the crack depending not only on their arrangement and dispersion but also on the variation of their material properties. The results of this study indicate that since CNTs behave as a barrier to crack growth. As a result, the benefits to clinical application are of significant importance.

\section{REFERENCES}

[1] G. Lewis and Y. Li, "Dependence of in Vitro Fatigue
Properties of PMMA Bone Cement on the Polydispersity Index of Its Powder," Journal of the Mechanical Behavior of Biomedical Materials, Vol. 3, No. 1, 2010, pp. 94-101. doi:10.1016/j.jmbbm.2009.05.003

[2] M. Nakai, M. Niinomi, T. Akahori, H. Tsutsumi, S. Itsuno, N. Haraguchi, Y. Itoh, T. Ogasawara, T. Onishi and T. Shindoh, "Development of Biomedical Porous Titanium Filled with Medical Polymer by in-Situ Polymerization of Monomer Solution Infiltrated into Pores,” Journal of the Mechanical Behavior of Biomedical Materials, Vol. 3, No. 1, 2010, pp. 41-50. doi:10.1016/j.jmbbm.2009.03.003

[3] R. Ormsby, T. McNally, C. Mitchell and N. Dunne, "Incorporation of Multiwalled Carbon Nanotubes to Acrylic Based Bone Cements: Effects on Mechanical and Thermal Properties," Journal of the Mechanical Behavior of Biomedical Materials, Vol. 3, No. 2, 2010, pp. 136-145. doi:10.1016/j.jmbbm.2009.10.002

[4] R. Ormsby, T. McNally, C. Mitchell and N. Dunne, "Influence of Multiwall Carbon Nanotube Functionality and Loading on Mechanical Properties of PMMA/MWCNT Bone Cements," Journal of Materials Science: Materials in Medicine, Vol. 21, No. 8, 2010, pp. 2287-2292. doi:10.1007/s10856-009-3960-5

[5] B. Marrs, R. Andrews and D. Pienkowski, "Multiwall Carbon Nanotubes Enhance the Fatigue Performance of Physiologically Maintained Methyl Methacrylate-Styrene Copolymer," Carbon, Vol. 45, No. 10, 2007, pp. 20982104. doi:10.1016/j.carbon.2007.05.013

[6] G. Lewis, "Properties of Acrylic Bone Cement: State of the Art Review," Journal of Biomedical Materials Research, Vol. 38, No., 1997, pp. 155-182. doi:10.1002/(SICI)1097-4636(199722)38:2<155::AID-JB M10>3.0.CO;2-C

[7] N. Ihaddadene, P. Erani, M. Cotifava, F. Tomei, M. Baleani, M. Viceconti, S. Bouzid and L. Cristofolini, "FatigueFractured Surfaces of Commercial Bone Cements," Computer Methods in Biomechanics and Biomedical Engineering, Vol. 10, Suppl. 1, 2007, pp. 157-158. doi:10.1080/10255840701479610

[8] J. Jeffers, M. Browne, A. Lennon, P. Prendergast and M. Taylor, "Cement Mantle Fatigue Failure in Total Hip Replacement: Experimental and Computational Testing," Journal of Biomechanics, Vol. 40, No. 7, 2007, pp. 15231533.

[9] B. A. O. McCormack and P. J. Prendergast, "Microdamage Accumulation in the Cement Layer of Hip Replacements under Flexural Loading," Journal of Biomechanics, Vol. 32, No. 5, 1999, pp. 467-476.

doi:10.1016/S0021-9290(99)00018-4

[10] M. Jasty, W. J. Maloney, C. R. Bragdon, D. O. O’Conner, T. Haire and W. H. Harris, "The Initiation of Failure in Cemented Femoral Components of Hip Antroplasties," Journal of Bone and Joint Surgery, Vol. 73B, No. 4, 1991, pp. 551-558.

[11] R. J. Kane, W. Yue, J. J. Mason and R. K. Roeder, “Improved Fatigue Life of Acrylic Bone Cements Reinforced with Zirconia Fibers," Journal of the Mechanical Behavior of Biomedical Materials, Vol. 3, No. 7, 2010, pp. 
504-511. doi:10.1016/j.jmbbm.2010.05.007

[12] K.-T. Lau and D. Hui, "The Revolutionary Creation of New Advanced Materials-Carbon Nanotube Composites,” Composites Part B: Engineering, Vol. 33, No. 4, 2002, pp. 263-277. doi:10.1016/S1359-8368(02)00012-4

[13] K. I. Tserpes, P. Papanikos and S. A. Tsirkas, "A Progressive Fracture Model for Carbon Nanotubes," Composites: Part B: Engineering, Vol. 37, No. 7-8, 2006, pp. 662-669. doi:10.1016/j.compositesb.2006.02.024

[14] V. A. Buryachenko and A. Roy, "Effective Elastic Moduli of Nanocomposites with Prescribed Random Orientation of Nanofibers," Composites Part B: Engineering, Vol. 36, No. 5, 2005, pp. 405-416. doi:10.1016/j.compositesb.2005.01.003

[15] W. Wei, A. Sethuraman, C. Jin, N. A. Monteiro-Riviere and R. J. Narayan, "Biological Properties of Carbon Nanotubes,” Journal of Nanoscience and Nanotechnology, Vol. 7, No. 4-5, 2007, pp. 1284-1297. doi:10.1166/jnn.2007.655

[16] S. K. Smart, A. I. Cassady, G. Q. Lu and D. J. Martin, "The Biocompatibility of Carbon Nanotubes," Carbon, Vol. 44, No. 6, 2006, pp. 1034-1047. doi:10.1016/j.carbon.2005.10.011

[17] L. P. Zanello, B. Zhao, H. Hu and R. C. Haddon, "Bone Cell Proliferation on Carbon Nanotubes," Nano Letters, Vol. 6, No. 3, 2006, pp. 562-567. doi:10.1021/nl051861e

[18] B. Zhao, H. Hu, S. K. Mandal and R. C. Haddon, “A Bone Mimic Based on the Self-Assembly Of Hydroxyapatite on Chemically Functionalized Single-Walled Carbon Nanotubes," Chemical Materials, Vol. 17, No. 12, 2005, pp. 3235-3241. doi:10.1021/cm0500399

[19] K. PourAkbar Saffar, A. R. Arshi, N. Jamilpour, A. Raeisi Najafi, G. Rouhi and L. Sudak, "A Cross-Linking Model for Estimating Young's Modulus of Artificial Bone Tissue Grown on Carbon Nanotube Scaffold,” Journal of Biomedical Materials Research Part A, Vol. 94A, No. 2, 2010, pp. 594-602. doi:10.1002/jbm.a.32737

[20] A. A. White, S. M. Best and I. A. Kinloch, "Hydroxyapatite-Carbon Nanotube Composites for Biomedical Applications: A Review," International Journal of Applied Ceramic Technology, Vol. 4, No. 1, 2007, pp. 1-13. doi:10.1111/j.1744-7402.2007.02113.X

[21] Y. Chen, Y. Q. Zhang, T. H. Zhang, C. H. Gan, C. Y. Zheng and G. Yu, "Carbon Nanotube Reinforced Hydroxyapatite Composite Coatings Produced through Laser Surface Alloying," Carbon, Vol. 44, No. 1, 2006, pp. 3745. doi:10.1016/j.carbon.2005.07.011

[22] B. Marrs, R. Andrews, T. Rantell and D. Pienkowski, "Augmentation of Acrylic Bone Cement with Multiwall Carbon Nanotubes," Journal of Biomedical Materials Research Part A, Vol. 77, No. 2, 2006, pp. 269-276. doi:10.1002/jbm.a.30651

[23] A. A. Gawandi, J. M. Whitney, R. B. Brockman and G. P. Tandon, "Interaction between a Nanofiber and an Arbitrarily Oriented Crack," Journal of Composite Materials, Vol. 42, No. 1, 2008, pp. 45-68.

[24] A. A. Gawandi, J. M. Whitney, G. P. Tandon and R. B. Brockman, "Three-Dimensional Analysis of the Interac- tion between a Matrix Crack and Nanofiber," Composites Part B: Engineering, Vol. 40, No. 8, 2009, pp. 698-704. doi:10.1016/j.compositesb.2009.04.001

[25] C. Atkinson, "On the Stress Intensity Factors Associated with the Cracks Interacting with an Interface between Two Elastic Media,” International Journal of Engineering Science, Vol. 13, No. 5, 1975, pp. 487-504. doi:10.1016/0020-7225(75)90018-X

[26] A. Romeo and R. A. Ballarini, "A Crack Very Close to a Bimaterial Interface,” ASME Journal of Applied Mechanics, Vol. 62, No. 3, 1995, pp. 614-619. doi:10.1115/1.2895990

[27] Z. Li and L. Yang, "The Near-Tip Stress Intensity Factor for a Crack Partially Penetrating an Inclusion,” Journal of Applied Mechanics, Vol. 71, No. 4, 2002, pp. 465-469. doi:10.1115/1.1651539

[28] P. S. Steif, “A Semi-Infinite Crack Partially Penetrating a Circular Inclusion,” Journal of Applied Mechanics, Vol. 54, No. 1, 1987, pp. 87-92. doi:10.1115/1.3172999

[29] A. Raeisi Najafi, A. R. Arshi, M. R. Eslami, S. Fariborz and M. H. Moeinzadeh, "Haversian Cortical Bone Model with Many Radial Microcracks: An Elastic Analytic Solution," Medical Engineering and Physics, Vol. 29, No. 6, 2007, pp. 708-717. doi:10.1016/j.medengphy.2006.08.001

[30] A. Raeisi Najafi, A. R. Arshi, K. PourAkbar Saffar, M. R. Eslami, S. Fariborz and M. H. Moeinzadeh, "A FiberCeramic Matrix Composite Material Model for Osteonal Cortical Bone Micromechanics Fracture: General Solution of Microcracks Interaction," Journal of the Mechanical Behavior of Biomedical Materials, Vol. 2, No. 3, 2009, pp. 217-223. doi:10.1016/j.jmbbm.2008.06.003

[31] N. A. Noda, Y. Takase and T. Hamashima, "Generalized Stress Intensity Factors in the Interaction within a Rectangular Array of Rectangular Inclusions," Archive of Applied Mechanics, Vol. 73, No. 5-6, 2003, pp. 311-322. doi:10.1007/s00419-002-0249-2

[32] Y. Qiao, X. Kong and E. Pan, "Fracture Toughness of Thermoset Composites Reinforced by Perfectly Bonded Impenetrable Short Fiber," Engineering Fracture Mechanics, Vol. 71, No. 18, 2004, pp. 2621-2633. doi:10.1016/j.engfracmech.2004.02.007

[33] M. B. Bush, "The interaction between a Crack and a Particle Cluster,” International Journal of Fracture, Vol. 88, No. 3, 1997, pp. 215-232. doi:10.1023/A:1007469631883

[34] K. Kim and L. J. Sudak, "Interaction between a Radial Matrix Crack and a Three-Phase Circular Inclusion with Imperfect Interface in Plane Elasticity,” International Journal of Fracture, Vol. 131, No. 2, 2005, pp. 155-172. doi:10.1007/s10704-004-3636-6

[35] P. G. Park and L. J. Sudak, "Stress Intensity Factor for an Interphase Crack Interacting with Two Imperfect Interfaces,” Mathematics and Mechanics of Solids, Vol. 15, No. 3, 2010, pp. 353-367. doi:10.1177/1081286508101512

[36] G. D. Seidel and D. C. Lagoudas, "Micromechanical Analysis of the Effective Elastic Properties of Carbon 
Nanotube Reinforced Composites," Mechanics of Materials, Vol. 38, No. 8-10, 2006, pp. 884-907. doi:10.1016/j.mechmat.2005.06.029

[37] J. P. Lu, "Elastic Properties of Carbon Nanotubes and Nanoropes,” Physical Review Letters, Vol. 79, No. 7, 1997, pp. 1297-1300. doi:10.1103/PhysRevLett.79.1297

[38] D. Swenson, M. James and B. Hardeman, "CASCA: A Simple 2-D Mesh Generator, Version 1. 4 User’s Guide,” Kansas State University, Manhattan, Kansas, 1997.

[39] P. Wawrzynek and A. Ingraffea, "FRANC2D: A TwoDimensional Crack Propagation Simulator,” Version 2. 7 User’s Guide, NASA CR 4572, 1994.
[40] X.-L. Xie, Y.-W. Mai and X.-P. Zhou, "Dispersion and Alignment of Carbon Nanotubes in Polymer Matrix: A Review," Materials Science and Engineering: R: Reports, Vol. 49, No. 4, 2005, pp. 89-112. doi:10.1016/j.mser.2005.04.002

[41] Y. Liu, C. Q. Ru, P. Schiavone and A. Mioduchowski, "New Phenomena Concerning the Effect of Imperfect Bonding on Radial Matrix Cracking in Fiber Composites,” International Journal of Engineering Science, Vol. 39, No. 18, 2001, pp. 2033-2050. doi:10.1016/S0020-7225(01)00049-0. 